\title{
Infrequent physeal wrist injury of the ulna and radius: a case report
}

\author{
Pedro Gutiérrez Carbonell · Eloisa Felíu Rey • \\ Javier Roca Vicente-Franqueira • \\ Antonio Perez Trigueros
}

Received: 16 April 2008/Accepted: 11 November 2008/Published online: 26 November 2008

(C) Springer-Verlag 2008

\begin{abstract}
We describe the case of a 13-year-old patient with a left wrist physeal fracture of the ulna and radius. X-Ray showed a Salter-Harris type II injury of the radius, and CT and MRI identified the following: type II epiphysiolysis of the radius with a metaphyseal wedge and type IV fracture of the ulna, with interposition in the focus of the fracture of the extensor carpi ulnaris tendon, as well as a tear in the central area of the triangular fibrocartilage complex. All these injuries were checked intraoperatively during open reduction. We believe that this clinical case illustrates the importance of carefully assessing physeal wrist injuries when they occur, especially in those cases involving the ulna and the radius. Diagnostic methods such as CT and MRI scans are indispensable for through evaluation and treatment of the injury.
\end{abstract}

Keywords Physeal ulna injury - TFCC injury in children $\cdot$ MRI in physeal wrist injury

P. G. Carbonell · J. R. Vicente-Franqueira · A. P. Trigueros Unit of Pediatric Traumatology and Orthopedics Pediatric Surgery, Hospital General Universitario de Alicante (Alicante University Hospital), Alicante, Spain

P. G. Carbonell - J. R. Vicente-Franqueira - A. P. Trigueros Department of Pathology and Surgery, "Miguel Hernandez" University, Elche, Spain

E. F. Rey

Radiology Department, Hospital General Universitario de Alicante (Alicante University Hospital), Alicante, Spain

P. G. Carbonell ( $\square)$

Paraje Ledua E-25, 03660 Novelda, Spain

e-mail: gutierrez_ped@gva.es

\section{Introduction}

Physeal fractures account for $18 \%$ of all fractures in children. Distal radius are $28 \%$ and distal ulna $3.3 \%$ of all physeal injuries. In $11-50 \%$ of the cases, distal radius fractures are associated to fractures of the ulna [1]. The results of conservative treatment (a plaster cast and closed reduction) are usually satisfactory. However, cases of socalled Galeazzi-equivalent fractures have been reported in children with wrist dorsiflexion and hyperpronation due to a fall on an outstretched hand. These cases have a poor prognosis and common sequelae such as early physeal closure of the ulna, bowing of the radius, limited range of movement (ROM) and loss of strength in the extremity. In a small percentage of these cases $(0.5-6.5 \%)$, the physeal injuries of the ulna are type IV Salter-Harris, with early physeal closure in 55\% of the cases [1-5]. On the other hand, early physeal closure of the radius after type II epiphysiolysis only amounts to $1-7 \%$ of the total [5]. Most authors recommend high-quality lateral and oblique $\mathrm{X}$-rays, as well as CT and/or MRI scans in order to diagnose soft-tissue injuries especially to the triangular fibrocartilage complex (TFCC) complex on the wrist [6-9].

\section{Case report}

This is a 13-year-old boy with trauma to his left arm due to hyperpronation after landing on his outstretched hand when he fell off of his bicycle. Upon examination, symptoms included pain, swollen and wrist deformity with normal neurovascular tests. X-rays revealed physeal fracture of the ulna and radius, more specifically, a Salter-Harris type II fracture of the radius and a type II or IV fracture of the ulna (Fig. $1 \mathrm{a}, \mathrm{b}$ ). Emergency closed reduction failed. Ct imaging 
Fig. 1 Anteroposterior (a) and lateral (b) X-rays showing radius fracture and displacement of the physeal fracture of the ulna
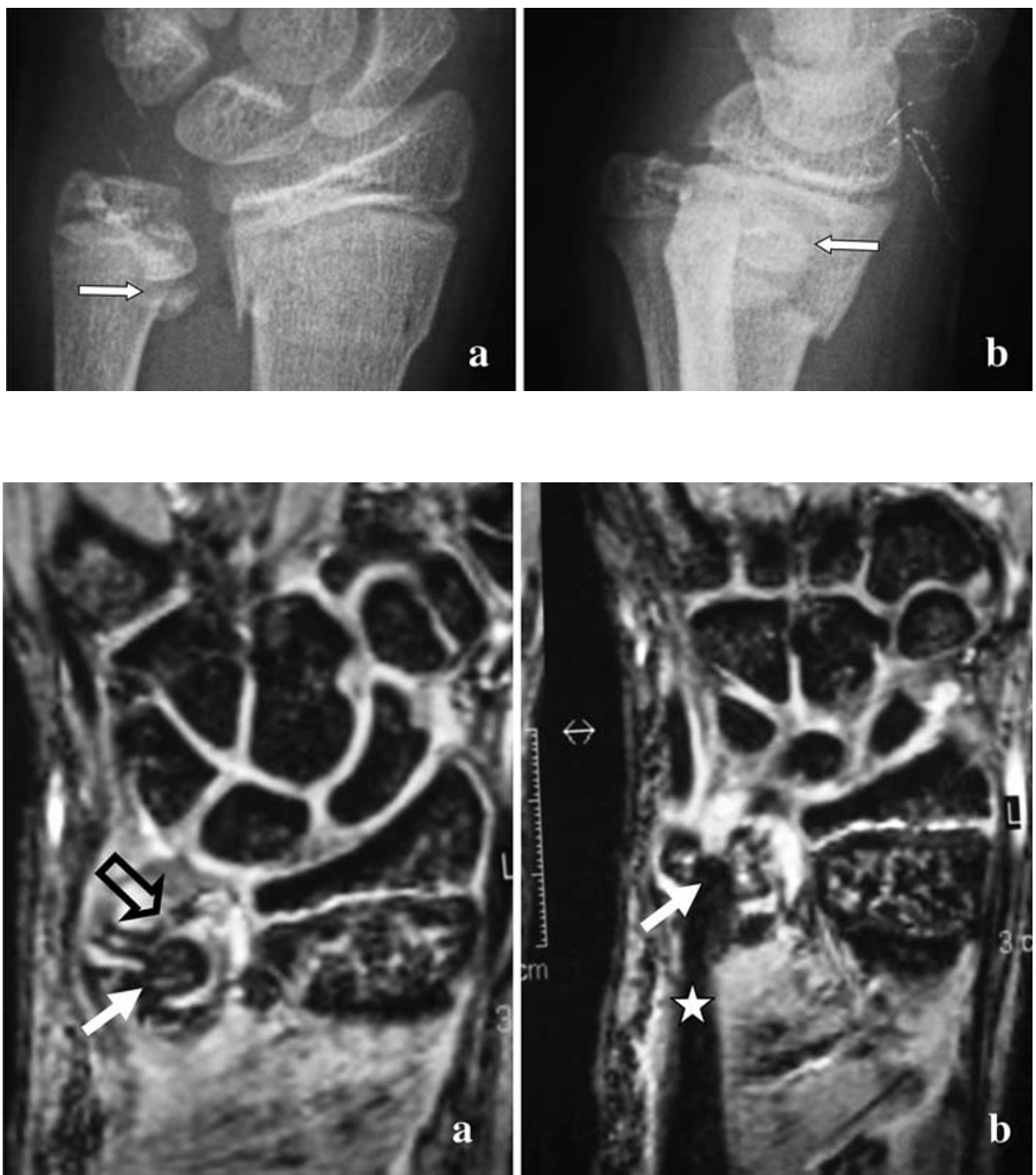

Fig. 2 a MRI (T2) in frontal plane, in which the tear of the carpal TFCC is marked with a hollow arrow, and the type IV epiphyseal fracture of the ulna is marked with a solid arrow. b MRI in which the ECU is marked with star and the ECU tendon that is interposed in the focus of the physeal injury of the ulna is marked with a solid arrow confirmed a metaphyseal fracture of the radius and a type IV Salter-Harris epiphyseal fracture of the ulna. MRI also confirmed: (a) injury of the radius with metaphyseal involvement not visible on the conventional X-ray, (b) a Salter type IV, Peterson type V fracture in ulna, (c) interposition of the extensor carpi ulnaris (ECU) at the point of ulnar fracture and (d) a Palmer type I B peripheral tear of the TFCC complex of the wrist (Fig. 2 a, b) $[1,5,10]$. Based on these findings, we performed an open reduction and fixed the fracture with K-wires. A long-arm cast was then put on with the forearm in the neutral position. During the procedure, all injuries were examined; reduction of the physeal fracture of the ulna was affected by the interposition of the ECU. The TFCC injury was sutured and reinforced with a retinaculum from the ECU [10]. Bone union was achieved with satisfactory alignment without pain (the patient plays the drums), but loss $10^{\circ}$ of pronation and supination range of movement (ROM). At last review (2 years follow-up) there was $0.8 \mathrm{~cm}$ of ulna minus configuration by ulna physeal arrest, but without distal bowing of the radius.

\section{Discussion}

$\mathrm{X}$-rays were previously used to diagnose these injuries [1113], but we believe MRI scans provide a more accurate picture of the anatomical disruption including the soft tissue components $[6,13]$. MRI scans allows us to distinguish between intrinsic and extrinsic wrist ligament injuries as well as those of the TFCC complex. MRI sensitivity is $89 \%$, specificity is $92 \%$ and diagnostic accuracy is $90 \%$, and comparable to invasive methods, such as arthroscopy and arthrogram, providing relevant information to therapeutic decision [6]. The TFCC can be better evaluated in the coronal plane from both $\mathrm{T} 1$ and especially $\mathrm{T} 2$, because the synovial fluid provides an "arthrogram" effect which makes the fracture easier to see. A normal TFCC in the coronal plane is seen as a structure with a hypointense, biconcave, triangular section between the lunar fossa of the radius and the styloid of the ulna. When a lesion exists, transverse fracture lines or shiny hyperintense signal perforations can be seen in the TFCC [7, 8]. These injuries are 
common (38-55\%) and of the degenerative type from the third decade of life, but are exceptional and of traumatic etiology in younger patients $[11,13,14]$. These traumatic lesions are misdiagnosed in around $41 \%$ of the cases, coinciding with physeal wrist injuries and resulting in instability, functional loss of ROM and ocassionaly important cosmetic alterations [4, 5, 14]. The closed reduction was impossible in this patient due to soft tissue interposition of the ECU and an open reduction was necessary before internal fixation [1, 11, 12, 15]. In these injuries at wrist joint, the information provided exclusively from conventional radiology is incomplete. We agree with other authors that pathological balance, and therefore correct treatment of the injuries is not possible except with the use of non-invasive diagnostic methods such as MRI scans, which should be obtained in cases in which there are possible physeal lesions of both the ulna and the radius $[3,6,9]$.

\section{References}

1. Peterson HA (2007) Epiphyseal growth plate fractures. Springer, Berlin

2. Golz RJ, Grogan DP, Greene TL, Belsole RJ, Orden JA (1991) Distal lunar physeal injury. J Pediatr Orthop 11:318-326

3. Peterson HA (1994) Physeal fractures: part 3. Classification. J Pediatr Orthop 14:439-448

4. Walsh HPJ, Aclaren CAN, Owen R (1987) Galeazzi fractures in children. J Bone Joint Surg 69B:730-733. doi:0301-620X/87/ $5145 \$ 2.00$
5. Waters PM, Bae DS, Montgomery KD (2002) Surgical management of posttraumatic distal radial growth arrest in adolescents. J Pediatr Orthop 22:717-724

6. Schneidmueller D, Maier M, Mack M, Straub R, Marzi I (2005) Therapeutische Relevanz der Magnetresonanztomographie bei Gelenkverletzungen im Kindesalter. Unfallchirurg 108:537-543. doi:10.1007/s00113-005-0936-x

7. Metz VM, Schratter M, Dock WI, Grabenwöger F, Kuzbari R, Lang S, Wanivenhaus AH, Puig S, Imhof H (1992) Age-associated changes of the triangular fibrocartilage of the wrist: evaluation of the diagnostic performance of MR imaging. Radiology 184:218-220

8. Skahen JR, Palmer AK, Levinsohn M, Buckingham SC, Szeverenyi NM (1990) Magnetic resonance imaging of the triangular fibrocartilage complex. J Hand Surg 15A:552-557

9. Zlatkin MB, Chao PC, Osterman AL, Schnall MD, Dalinka MK, Kressel HY (1998) Chronic wrist pain: evaluation with highresolution imaging. Radiology 173:723-729

10. Palmer AK (1989) Triangular fibrocartilage complex injuries: a classification. J Hand Surg 14A:594-606

11. Evans DL, Stauber M, Frykman GK (1990) Irreducible epiphyseal plate fracture of the distal ulna due to interposition of the extensor carpi ulnaris tendon. A case report. Clin Orthop 251:162-165

12. Faraj AA, Kumar MS, Ketzer B, Rawes M (2000) An irreducible Salter-Harris type IV distal ulna fracture. Injury 3:746-748

13. Kamano M, Ko H, Kazuki K (2005) Paediatric Galeazzi-equivalent fracture: two case reports. Hand Surg 10:249-254

14. Yamamoto K, Tateiwa T, Takaaki S, Miyajima H, Nagai S, Imakiire A (2006) Treatment of epiphyseal injury of the distal ulna without associated radial fracture. Orthopedics 29:157-159

15. Imatani J, Hashizume H, Nishida K, Morito Y, Inoue H (1996) The Galeazzi-equivalent lesion in children revisited. J Hand Surg 21B:455-457 